\title{
The imbalance of Th17/Treg cells is involved in the progression of nonalcoholic fatty liver disease in mice
}

Beihui He${ }^{1}$, Liyan Wu', Wei Xie ${ }^{2}$, Yitong Shao ${ }^{3}$, Jianping Jiang ${ }^{4}$, Zhenzhong Zhao ${ }^{5}$, Maoxiang Yan', Zhiyun Chen $^{1 *}$ and Dawei Cui ${ }^{6}$

\begin{abstract}
Background: Non-alcoholic fatty liver disease (NAFLD) is a common, chronic liver disease worldwide. Recent studies have shown that T helper (Th) 17 and regulatory T (Treg) cells play critical roles in various disorders of liver inflammation. Here, we explored the value of polyene phosphatidylcholine capsules (PPC) for regulating the imbalance of Th17/Treg cells in the pathogenesis of mice with NAFLD.

Methods: C57BL/6 mice were randomly divided into three groups as follows:normal diet (ND), high-fat diet (HF) , and HF plus PPC(HF + PPC). The frequencies of splenic Th17 and Treg cells were measured by flow cytometry, and their related cytokines were analyzed by CBA and real-time PCR.

Results: At the end of 24 weeks, mice in the HF group had a higher frequency of intrahepatic Th17 cells,and a lower proportion of Treg cells compared with the ND group. The levels of Th17 cell-related cytokines (IL-6, IL-17 and IL-23) in serum and in liver tisse were increased,and the hepatic mRNA levels of RORyt, STAT3 and IL-6 were also increased. By contrast,the FoxP3 mRNA level was decreased in the HF group. Moreover, significant pathological and biochemical changes in the liver, as well as serum biochemical changes, were found in mice with NAFLD. Interestingly, following treatment with PPC, the levels of liver inflammation,frequencies of Th17/Treg cells and associated cytokines,and biochemical data were significantly altered.
\end{abstract}

Conclusion: These findings demonstrate a critical role for PPC in partially attenuating liver inflammatory responses in mice with NAFLD that involves the imbalance of Treg/Th17 cells and associated cytokines.

Keywords: Nonalcoholic fatty liver disease, Th17 cells, Treg cells

\section{Background}

Non-alcoholic fatty liver disease (NAFLD),characterized by steatosis, lobular inflammation and hepatocellular ballooning,comprises a spectrum of liver disorders,including simple fatty liver,non-alcoholic steatohepatitis (NASH),hepatic fibrosis, and cirrhosis. NAFLD has become one of the most common chronic diseases worldwide,affecting approximately one-third of population [1-3]. Approximately one-third of patients with NASH have an increased risk of developing liver fibrosis, cirrhosis and hepatocellular

\footnotetext{
* Correspondence: zhiyunchen63@163.com

'Laboratory of Digestive Disease, the First Affiliated Hospital of Zhejiang Chinese Medical University, 54,Youdian Road, Hangzhou 310006, Zhejiang Province, People's Republic of China

Full list of author information is available at the end of the article
}

carcinoma [3, 4]. The mechanisms mediating these liver disorders,from simple liver steatosis to NASH,remain unclear. Lipopolysaccharide (LPS), oxidative stress, cytokines and other pro-inflammatory mediators may be involved in imposing a 'second hit' during the transitional process $[4,5]$. Hepatic inflammation is one of the most pronounced features of $\mathrm{NASH}$, and hepatic immune responses play critical roles in the pathogenesis of NASH and other progressive diseases $[5,6]$. It is believed that an imbalance between anti-inflammatory $\mathrm{T}$ helper type 2 (Th2) cytokines and pro-inflammatory Th1 cytokines is responsible for the development of NASH [6, 7].

Aside from the Th1/Th2 paradigm, recent studies have demonstrated the crucial impact of Th17 cells on hepatic inflammation $[8,9]$. Th17 cells express IL-17, IL- 
17 F, IL-21 and IL-22 cytokines and mediate potent inflammatory immune responses [10]. Accumulating evidence has shown that Th17 cells, and the associated cytokine IL17 , may promote a pro-inflammatory state in chronic viral hepatitis, autoimmune liver diseases, alcoholic liver disease and hepatocellular carcinoma [11-14].By contrast,Treg cells have anti-inflammatory functions and confer protection against liver inflammation $[12,13]$. The reciprocal relationship between Treg cells and Th17 cells represents a delicate balance between tolerance and elicitation of immune responses [12]. Previously, we had demonstrated that the proportion of Treg cells was significantly lower in patients with non-alcoholic liver disease [15]. Furthermore, we have focused on the imbalance between Treg and Th17 cells in the development of NAFLD by using a mouse model, which may provide significant understanding of NAFLDassociated inflammatory processes.

In this study, we report that the frequencies of Th17 cells in the high fat diet (HF) group were higher than those in the normal diet (ND) group. Conversely, the frequencies of Treg cells in the HF group were lower than those in the ND group. The levels of Th17 and Treg cell-associated cytokines in the serum and liver were changed in the HF group compared with the ND group. Additionally, hepatic mRNA levels of Th17 cellrelated genes, including ROR $\gamma \mathrm{t}$, STAT3 and IL-6, were increased,whereas the level of FoxP3 mRNA was decreased in the HF group. Moreover, significant pathological changes in the liver were found,and biochemical changes in the liver and sera were also observed in mice with NAFLD. Interestingly, after treatment with polyene phosphatidylcholine capsules (PPC), the liver inflammation,Th17/Treg cell frequencies,and biochemical data were significantly changed in this study. Taken together,an imbalance between Th17/Treg cells and associated cytokines may be involved in the pathological development of NAFLD, and PPC can attenuate the inflammatory response.

\section{Methods}

\section{Animal experiments}

A total of 80 male C57BL/6 mice (age 6 weeks, each weighing 18-20 g) were purchased from the Shanghai SLAC Laboratory Animal Co.,Ltd.(Shanghai, China) and fed under pathogen-free conditions in the animal facility of Zhejiang Chinese Medical University [certification NO. SCXK (HU) 2007-0005]. Similar to a previous report [3], mice were randomly divided into three treatment groups as follows: an ND group $(n=30)$ (ND, $8 \%$ rice bran, $51 \%$ maize, $30 \%$ soybean powder, $3 \%$ bone powder, $1.3 \%$ multivitamin, and $6.7 \%$ mineral), an HF group $(n=30) \quad(\mathrm{HF}, 75 \% \mathrm{ND}, 2 \%$ cholesterol, $15 \%$ lard and $8 \%$ yolk powder), and an HF + PPC group $(n=20)$. Mice in the HF + PPC group were fed the HF diet with an additional intragastric administration of PPC (PPC, $195.40 \mathrm{mg} / \mathrm{kg} / \mathrm{mouse}$, Sanofi, Beijing, China) at the 7th week. The mice were kept at room temperature $\left(21^{\circ} \mathrm{C}\right)$ in a light-controlled environment with ad libitum access to food and water. All animal experiments were approved by the Institutional Animal Care and Use Committee of the First Affiliated Hospital of Zhejiang Chinese Medical University and were conducted in accordance with the National Research Council Guide for Care and Use of Laboratory Animals. The blood was collected by retroorbital route under anesthesia.

\section{Pathological changes in mice with NAFLD}

To determine whether cellular and biochemical changes are consistent with the development of steatohepatitis and whether a possible reversal of the disease process may occur after PPC treatment, we randomly selected ten mice from the ND and HF groups at the 8th, 16th, and 24th weeks and randomly selected ten mice from the HF + PPC group at the 16th and 24th weeks. These mice were sacrificed to obtain liver and serum samples for further study. Liver tissues were stained either with H\&E stain (hematoxylin and eosin) or Masson's trichrome stain and subsequently observed using a light microscope (Olympus, CX41, Japan).

Treg and Th17 cell frequency analysis by flow cytometry Splenic lymphocytes from mice were isolated according to a previously reported method [14], and these cells were stained with APC-conjugated anti-mouse CD25 and FITC-conjugated anti-mouse CD4 or isotypes (eBioscience, USA) for $20 \mathrm{~min}$ at $4{ }^{\circ} \mathrm{C}$. These cells were washed twice, fixed, permeabilized and then stained with PE-conjugated anti-mouse Foxp3 for analysis of Treg subpopulations. To detect splenic Th17 cells, splenocytes were stimulated with $50 \mathrm{ng} / \mathrm{ml}$ phorbol 12-

Table 1 Primer sequences of T helper type 17 (Th17)-related mouse cytokines

\begin{tabular}{llll}
\hline Name & & Primers $\left(5^{\prime} \rightarrow 3^{\prime}\right)$ & Product (bp) \\
\hline B-actin & Sense & AGAGGGAATCGTGCGTGAC & $138 \mathrm{bp}$ \\
& Antisense & CAATAGTGATGACCTGGCCGT & \\
IL-6 & Sense & ACAACCACGGCCTTCCCTACT & $139 \mathrm{bp}$ \\
& Antisense & CACGATtTCCCAGAGAACATGTG & \\
RORyt & Sense & GCCGCGGAGCAGACACACT & $176 \mathrm{bp}$ \\
& Antisense & GGAGGCCCCCTGGACCTCTG & \\
Foxp3 & Sense & CAGGAGAAAGCGGATACCAAATG & $176 \mathrm{bp}$ \\
& Antisense & ATCTGTGAGGACTACCGAGCC & \\
STAT3 & Sense & ACCTCCAGGACGACTTGAT & $203 \mathrm{bp}$ \\
& Antisense & TGTCTTCTGCACGTACTCCA & \\
\hline
\end{tabular}

The housekeeping gene $\beta$-action was used as an internal control, and genespecific mRNA expression was normalized against $\beta$-action expression. Relative quantification by the $2^{-\Delta \Delta \mathrm{Ct}}$ method was realized by comparing to control groups 
myristate 13-acetate (PMA) (BioVision, Mountain View, CA, USA), $1 \mu \mathrm{g} / \mathrm{ml}$ ionomycin (Enzo Life Sciences, Farmingdale, NY, USA) and $500 \mathrm{ng} / \mathrm{ml}$ monensin (eBscience, San Diego, CA, USA) for $4 \mathrm{~h}$ and were stained with FITC-conjugated anti-mouse CD4 and PEconjugated anti-mouse IL-17 antibodies. Acquisitions were performed using a FACS Canto II flow cytometer (BD, USA). Data were analyzed based on the percentages of Th17 cells and Treg cells.

\section{Serum biochemistry and cytokines of mice with NAFLD}

Serum from mice was collected at 8, 16 and 24 weeks. Alanine aminotransferase (ALT), aspartate aminotransferase (AST), triglyceride (TG) and total cholesterol (CHOL) levels were measured using a multi-channel auto-analyzer (Hitachi 7180, Japan). Levels of serum cytokines, including TNF- $\alpha$, IL-6, IL-17, IL-23, and TGF- $\beta$, were also measured by ELISA (eBioscience, San Diego, USA).
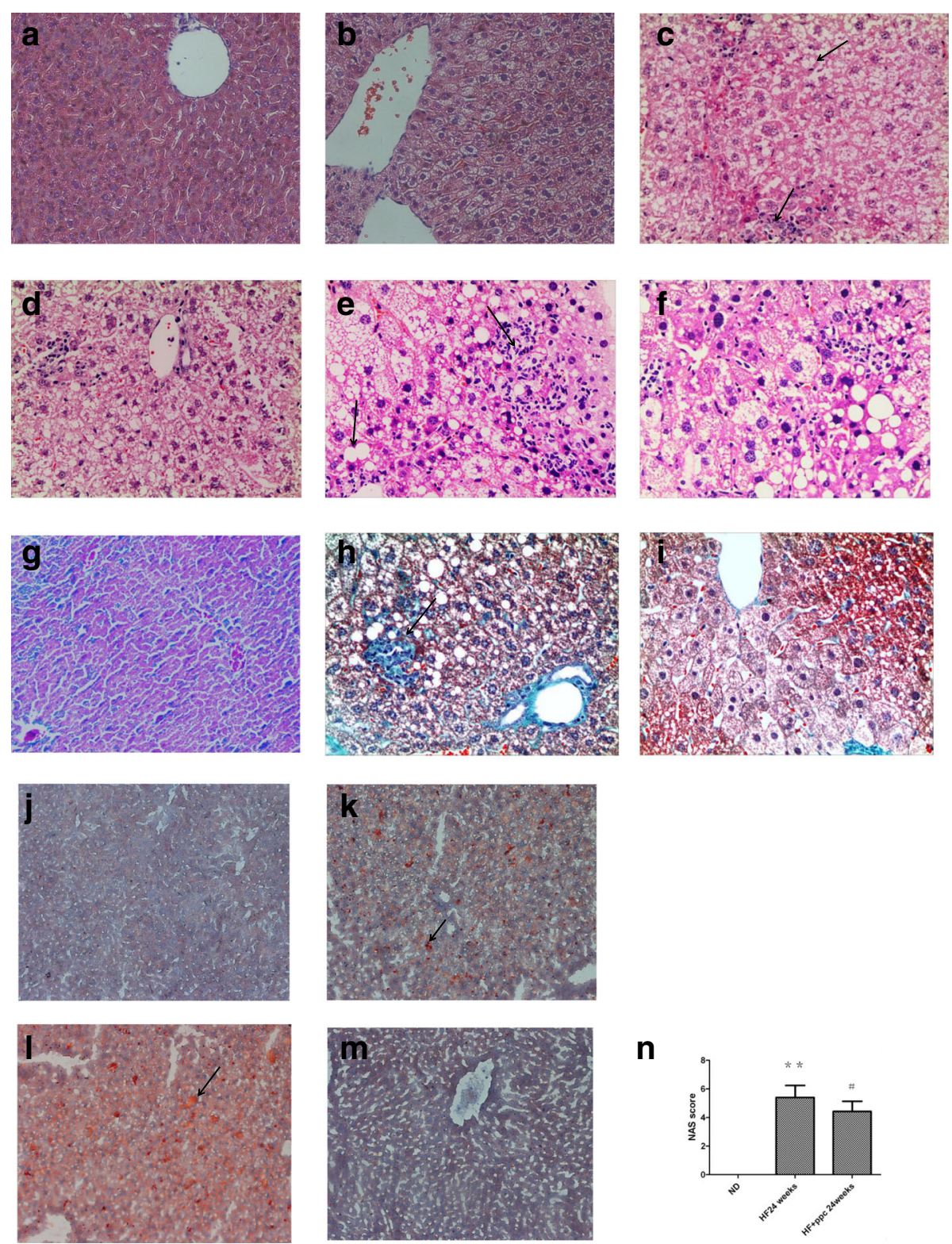

Fig. 1 Pathologic changes of liver tissue in mice. a ND group at 8 weeks; $\mathbf{b}$ HF group at 8 weeks; $\mathbf{c}$ HF group at 16 weeks; $\mathbf{d}$ HF + PPC group at 16 weeks; e HF group at 24 weeks; $\mathbf{f}$ HF + PPC group at 24 weeks; $\mathbf{g}$ ND group at 24 weeks; $\mathbf{h}$ HF group at 24 weeks; $\mathbf{i}$ HF + PPC group at 24 weeks; $\mathbf{j}$ ND group at 8 weeks; $\mathbf{k}$ HF group at 8 weeks; I HF group at 16 weeks; $\mathbf{m}$ HF + PPC group at 16 weeks; $\mathbf{n}$ NAS score (a-f, H\&E stain, magnification $\times 200$; $\mathbf{g}$-i Masson's trichrome staining, magnification $\times 200$; j-m Oil Red O staining,magnification $\times 100) . \mathbf{n} \mathrm{HF}$ vs. ND: ${ }^{* *}, P<0.01$. HF + PPC vs. HF: \#, $P<0.05$ 


\section{RNA extraction and real-time PCR assay}

RNA was extracted from the liver of mice using Trizol reagent, and complementary DNA synthesis was performed according to the manufacturer's instructions. PCR assays were conducted using an ABI7900 Real Time PCR machine and SYBR Premix Ex TaqTM (Takara, Shiga, Japan). The primer sequences of IL-6, retinoid-related orphan receptor RORyt, STAT3, FoxP3 and $\beta$-actin genes are presented in Table 1.

\section{Statistical analysis}

Data are expressed as the means and standard deviations (mean \pm SD). Data were analyzed by ANOVA. All statistical analyses were performed using the statistical SPSS software (version 17.0, SPSS Inc., USA). P-values $<0.05$ were considered statistically significant.

\section{Results}

Pathologic changes of liver tissue in NAFLD mice

Compared with the ND group, moderate liver cell steatosis and mild lobular inflammatory cell infiltration were observed in mice from the HF group at 8 weeks (Fig. 1a and b). At 16 weeks, severe fatty degeneration, lobular infiltration of inflammatory cells and significantly increased focal necrosis were found in mice from the HF group compared with that at 8 weeks (Fig. 1c and d). The mice in the HF group developed severe steatosis, further increased lobular inflammation and necrosis, and perisinusoidal fibrosis of varying degrees at 24 weeks (Fig. 1e and g). After treatment with PPC, murine liver cells displayed a lower degree of inflammation despite the mice still being fed a high-fat diet (Fig. 1f and i). The average NAS scores were significantly reduced in $\mathrm{HF}+$ PPC group at 24 weeks $(P<0.05)$ (Fig. 1 n). Oil red $\mathrm{O}$

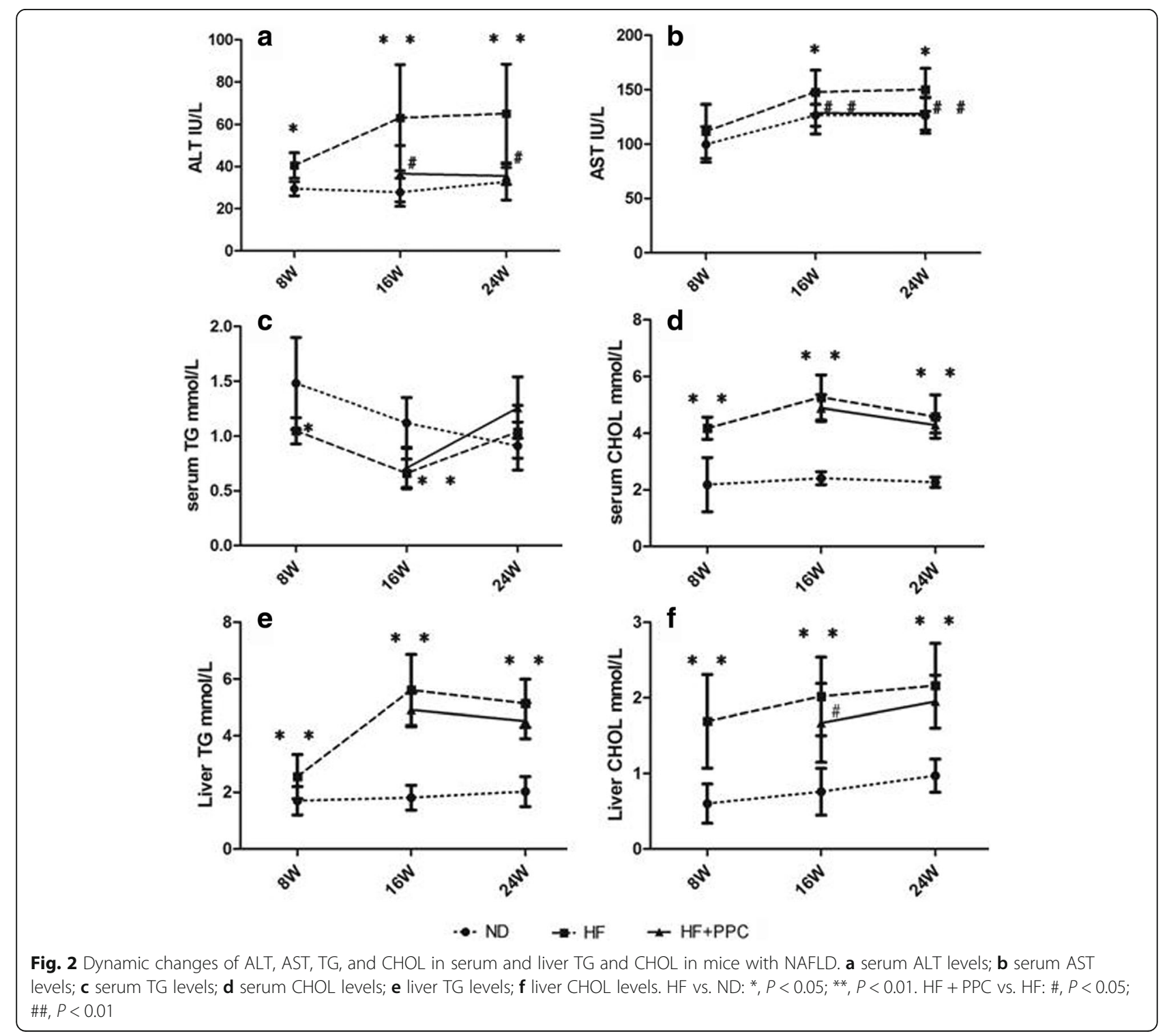


staining provided a much more indicative view of the fat accumulation in HF groups at 8 and 16 weeks,and the fat accumulation could be significantly ameliorated by PPC treatment (Fig. 1j, k, l and $\mathrm{m}$ ). Therefore, microscopic pathological changes in line with NASH were observed in mice fed a high-fat diet, and these pathological changes inflammatory changes could be significantly ameliorated by PPC treatment.

\section{Biochemical changes in serum and liver in NAFLD mice}

Serum AST levels from mice in the HF group were higher at 8 weeks than those in the ND group $(P<0.05)$ and were dramatically increased at 16 and 24 weeks compared with the ND group $(P<0.01)$. An increasing trend in the serum ALT level was observed in the HF group at 8 weeks, although there was no statistically significant difference between the HF and ND groups $(P>0.05)$. Serum ALT levels in the HF group were increased at both 16 and 24 weeks compared with those in the ND group $(P<0.05)$. After the intervention with PPC, at 16 and 24 weeks, both the AST and ALT levels were remarkably decreased compared with the HF group $(P<0.05$ and $P<$ 0.01 , respectively) (Fig. $2 \mathrm{a}$ and b). The serum $\mathrm{CHOL}$ and liver TG levels in the HF group at 8, 16 and 24 weeks were significantly higher than those in the ND group $(P<0.01)$, with a peak at 16 weeks (Fig. $2 \mathrm{~d}$ and e). The serum TG levels in the HF group at 8 and 16 weeks were decreased in contrast to those in the ND group $(P<0.05$ and $P<$
0.01 , respectively) (Fig. 2c). After treatment with PPC, the liver $\mathrm{CHOL}$ levels in mice at 16 weeks notably declined $(P<0.05)$, whereas no significant difference was observed at 8 and 24 weeks compared with the HF group $(P>0.05)$ (Fig. 2f).

\section{Frequencies of Treg cells and Th17 cells in NAFLD mice} The frequencies of splenic Treg cells and Th17 cells in mice with NAFLD were analyzed by flow cytometry (Fig. 3a). The frequencies of splenic Th17 cells in the HF group were significantly increased compared with the ND group at 24 weeks $(P<0.05)($ Fig. $3 b)$. Treg cells had a tendency to decrease at 24 weeks, but no statistical significance was observed $(P>0.05)$ (Fig. 3b). After the intervention with $\mathrm{PPC}$, we observed a decrease in the Th17 cell population and an increase in the Treg cell population in the $\mathrm{HF}+\mathrm{PPC}$ group compared with the HF group at 24 weeks, but these trends were not significant $(P>0.05)$ (Fig. 3a and b).

\section{Serum cytokines in NAFLD mice}

Cytokine (TNF- $\alpha$, IL-6, IL-17, and IL-23) levels gradually increased from 8 to 24 weeks in the HF group (Fig. 4). The levels of the aforementioned cytokines in the HF group were not significantly different compared with the ND group at 8 weeks $(P>0.05)$, whereas the levels at 16 and 24 weeks were significantly increased compared with the ND group $(P<0.05$ and $P<0.01$, respectively $)$

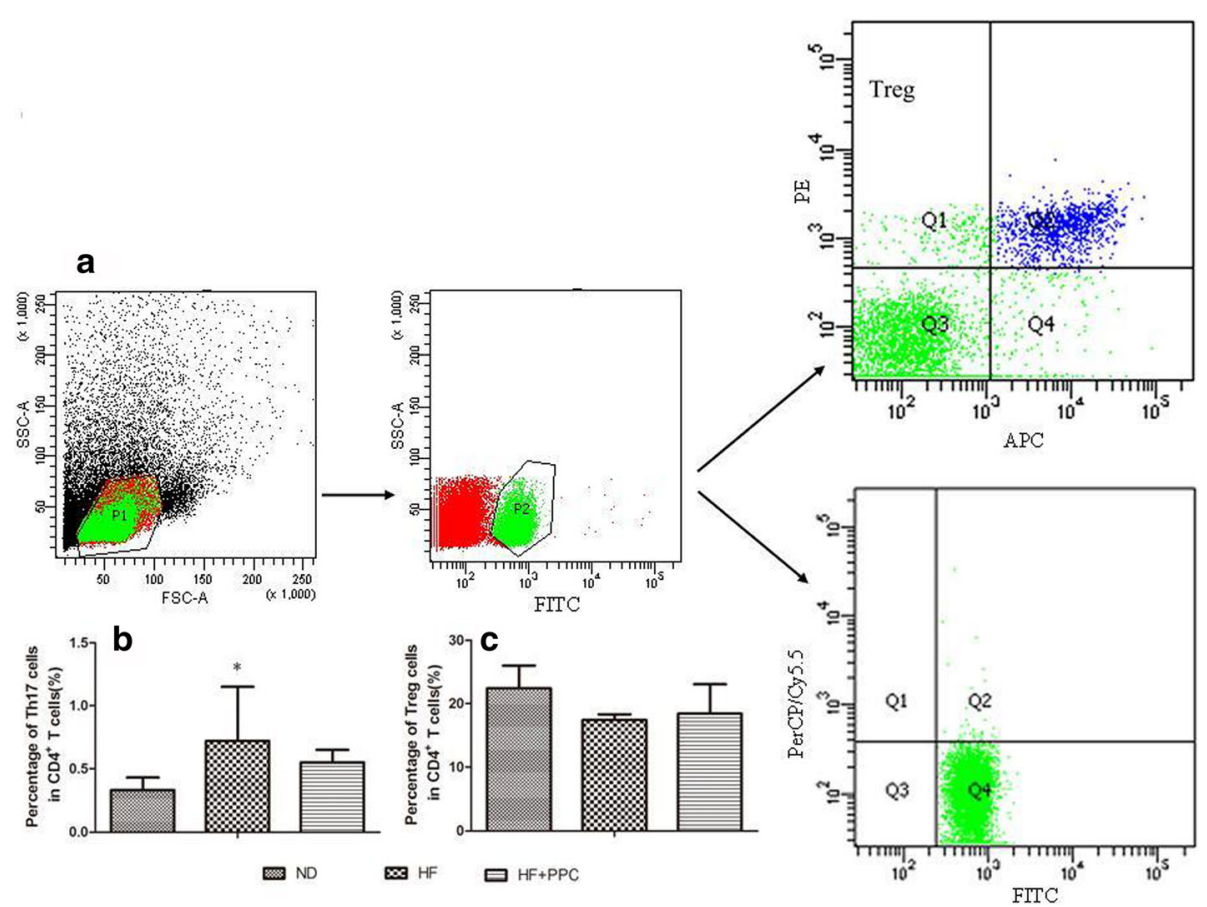

Fig. 3 Frequency changes of splenic Treg and Th17 cells in mice with NAFLD. a frequencies of Treg and Th17 cells were detected by flow cytometry; b frequency of Th17 cells; c frequency of Treg cells in the three groups. HF vs. ND: ${ }^{*}, P<0.05 ;{ }^{*}, P<0.01$. HF + PPC vs. HF: \#, $P<0.05 ; \# \#, P<0.01$ 

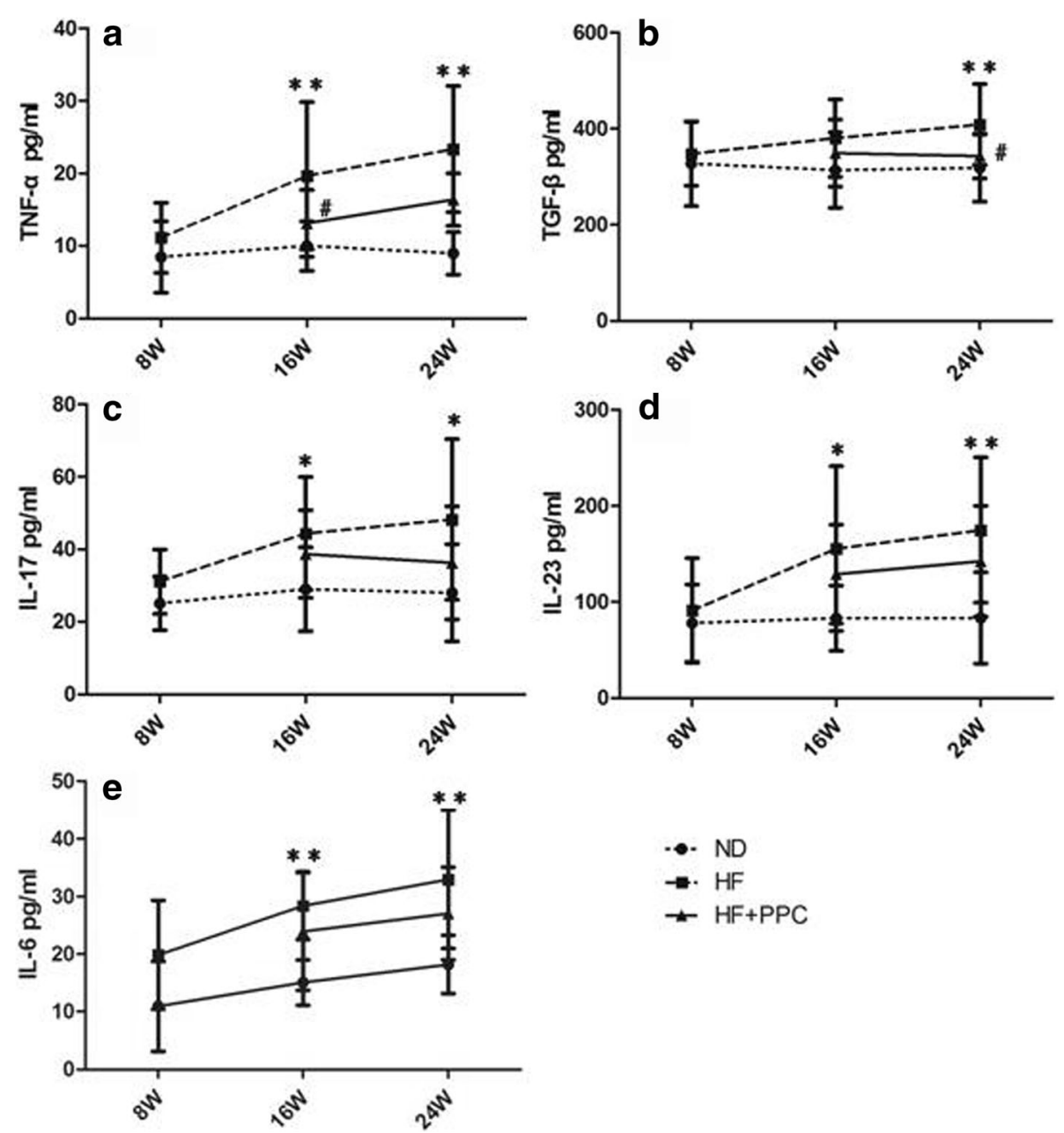

Fig. 4 Dynamic changes of Th17 and Treg cell-related cytokines in serum in mice with NAFLD. a serum TNF-a levels; b serum TGF- $\beta$ levels; $\mathbf{c}$ serum IL-17 levels; d serum IL-23 levels; e serum IL-6 levels. HF vs. ND: ${ }^{*}, P<0.05 ;{ }^{*}, P<0.01$. HF + PPC vs. HF: \#, $P<0.05 ; \# \#, P<0.01$

(Fig. 4a and c-e). The TGF- $\beta$ levels in the HF group were only significantly higher at 24 weeks compared with the ND group $(P<0.01)$ (Fig. 4b). After the intervention with PPC, the TNF- $\alpha$ levels in the HF + PPC group at 16 and 24 weeks were significantly decreased compared with the HF group $(P<0.05)$ (Fig. 4a). Meanwhile, the TGF- $\beta$ levels in the HF + PPC group at 24 weeks were significantly decreased compared with the HF group ( $P$ $<0.05$ ) (Fig. 4b); however, other cytokines showed no significant difference $(P>0.05)$ (Fig. 4c-e).

\section{Treg/Th17-related mRNA expression in the liver tissues of NAFLD mice}

The RORyt, STAT3, and IL-6 mRNA levels and ROR $\gamma \mathrm{t}$ / FoxP3 ratio in the HF group were not significantly different compared with the ND group at 8 weeks $(P>0.05)$; however, they significantly increased compared with the ND group at 16 and 24 weeks $(P<0.05$ and $P<0.01$, respectively). The expression levels of ROR $\gamma$ t, STAT3, and
IL-6 mRNA and RORyt/FoxP3 ratio in the $\mathrm{HF}+\mathrm{PPC}$ group at 24 weeks were significantly decreased compared with the HF group $(P<0.05)$ (Fig. 5a, b, c and e). The expression of FoxP3 mRNA in the liver tissues in the HF group at 16 weeks was significantly decreased $(P<0.05)$, but no significant difference at 8 or 24 weeks was observed compared with the ND group $(P>0.05)$ (Fig. $5 \mathrm{~d})$.

\section{Discussion}

Accumulating evidence indicates that approximately $30 \%$ of NAFLD patients may progress to NASH, which is characterized by lobular inflammation and ballooning degeneration, and these patients may further develop liver fibrosis, liver cirrhosis and hepatocellular carcinoma [16]. Understanding the mechanisms by which simple fatty liver develops into NASH is critical. Several risk factors (such as LPS, oxidative stress and inflammatory cytokines) are likely to provide a "second hit", which may contribute to the transformation process from simple 

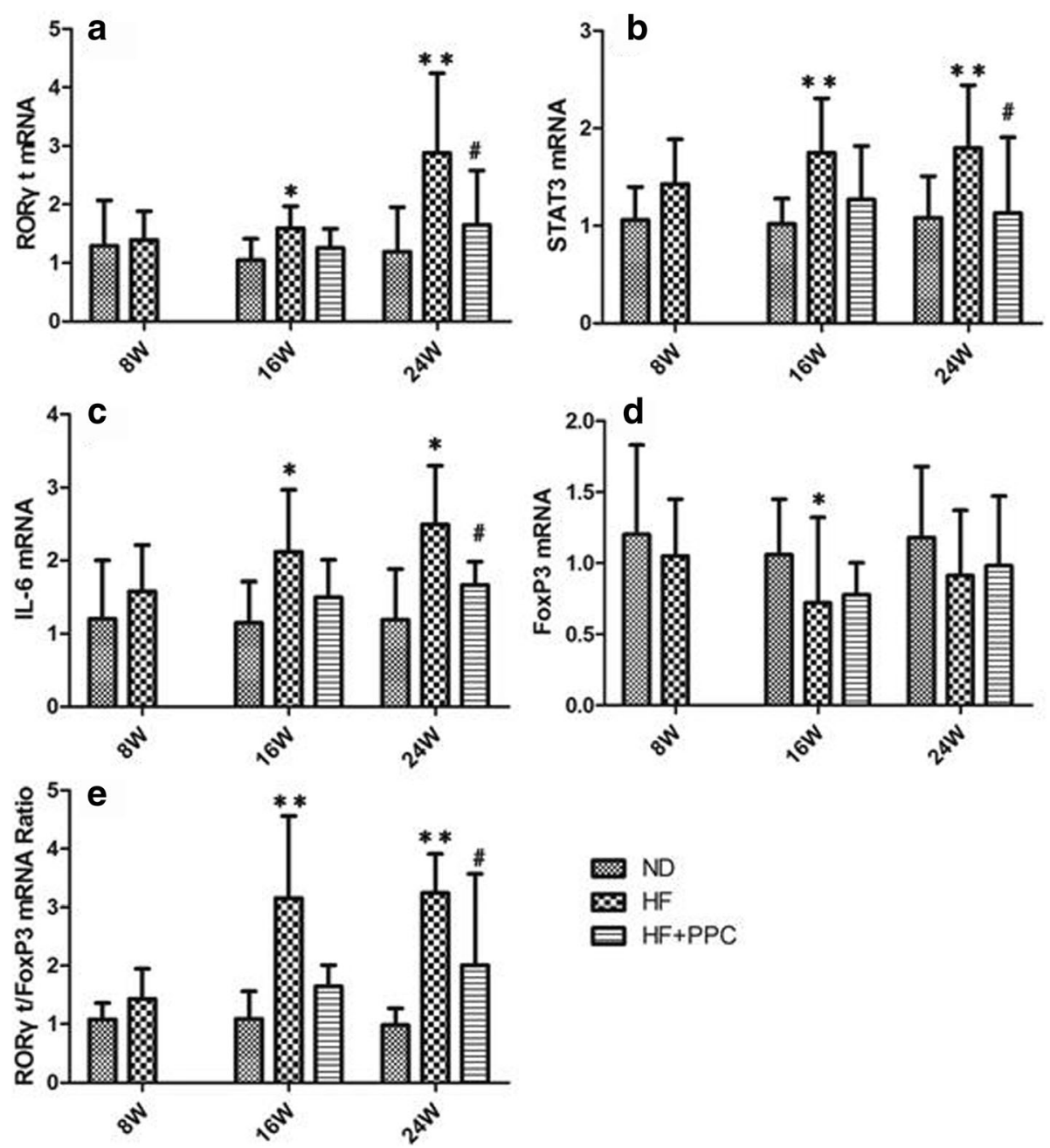

Fig. $\mathbf{5}$ Increased mRNA expression of IL-6, RORYt, STAT3, and FoxP3 in mice with NAFLD. a levels of the relative expression of RORyt mRNA in liver; $\mathbf{b}$ levels of the relative expression of STAT3 mRNA in liver; $\mathbf{c}$ levels of the relative expression of IL-6 mRNA in liver; $\mathbf{d}$ levels of the relative expression of FoxP3 mRNA in liver; e ratio of RORyt/FoxP3 mRNA in liver from mice with NAFLD. HF vs. ND: ${ }^{*}, P<0.05 ;{ }^{* *}, P<0.01$. HF + PPC vs. HF: \#, $P<0.05 ; \# \#, P<0.01$

steatosis to NASH $[4,5,17]$. Several studies have demonstrated that immune disorders, such as an imbalance of anti-inflammatory cytokines and pro-inflammatory cytokines, are involved in liver inflammation $[18,19]$. A previous study suggested that NASH is mainly associated with an imbalance of Th1/Th2 immune responses, with Th1 predominance [20].

Recent reports suggest that Th17 cells, which produce IL-17A, IL-17 F, IL-21 and IL-22, not only mediate the clearance of pathogens but also promote the progression of tissue inflammation and autoimmune diseases [21, 22]. IL- 6 and TGF- $\beta$ cytokines are required for differentiation and proliferation of Th17 cells expressing the transcription factor RORyt. IL-21 and IL-23 cytokines contribute to the differentiation of Th17 cells, and IL-23 promotes maturation in the later stages of differentiation of Th17 cells [20-23]. IL-17, IL-6 and IL-21 cytokines can aggravate the development of autoimmune disease by triggering inflammatory reactions [20, 24]. IL-6 was demonstrated to paralyze the Treg cells' suppressive effects on Th17 cells and contributed to liver injury by promoting expansion of Th17 cells [24]. STAT3 and RORyt are specific transcription factors responsible for the differentiation of Th17 cells, and the expression of IL-17 mRNA is closely associated with RORyt expression [22]. Treg cells can inhibit inflammation and maintain self-tolerance, and an altered balance of TGF- $\beta$ and IL- 6 may control the switching of Treg or Th17 cells via antagonistic competition of Foxp3 and RORyt [25-27].

The functions of Th17 cells and IL-17 in liver diseases have been extensively explored [13, 26, 28]. Th17 cells are associated with hepatocellular steatosis and inflammatory processes [13]. IL-17 and fatty acids can synergistically stimulate hepatic cells to secrete IL-6, and IL-6 
aggravates insulin resistance. As IL-17 increases hepatic inflammation, their cooperation can exacerbate the development of NAFLD $[8,29]$. By contrast, $\mathrm{CD} 4^{+} \mathrm{CD} 25$ ${ }^{+}$Foxp $3{ }^{+}$Treg cells contribute to attenuation of liver inflammatory diseases [30, 31]. Previous studies have shown that the number of hepatic Treg cells was significantly decreased in mice with NAFLD [8], and the percentage of Treg cells and expression of Foxp3 mRNA were also decreased in mice with NASH [32]. Our previous report indicated that the proportion and number of Treg cells were significantly lower in NAFLD patients [15]. Importantly, the Treg/Th17 balance was relevant for NAFLD, and it may predict long-term outcomes in NAFLD patients. In this study, we found that NAFLD mice experienced a transformation process from simple fatty liver disease to fatty hepatitis to the early stages of liver fibrosis. The frequencies of Th17 cells were elevated, with increased mRNA levels of IL-6, RORyt, and STAT3 genes in liver tissues and high concentrations of IL-17 in serum. These findings are in accordance with previous reports $[8,29-32]$. PPC, trade name Essentiale ${ }^{\circ}$, can partially alleviate liver injury, improve liver enzyme activity, promote the regeneration of liver tissue, and is commonly used in the clinical treatment of NAFLD [15]. In this study, liver inflammation and fibrosis of varying degrees were reduced after treatment with PPC at 16 and 24 weeks. The mRNA expression levels of RORyt, STAT3, and IL-6 genes and the RORyt/FoxP3 ratio were significantly reduced at 24 weeks compared with the HF group. The imbalance of Treg/Th17 also had a distinct correction. According to these findings, we hypothesize that under normal conditions, the ratio of Treg/Th17 cells in the host is balanced. A long-term high-fat diet causes abnormal secretions of murine liver tissue cytokines TGF- $\beta$ and IL- 6 that co-stimulates the further activation of STAT3 and RORyt expression. This process promotes the differentiation of CD4 + T cells to Th17 cells and the release of IL-17, and Th17 cells inhibit the differentiation of Treg cells. The more dominant Th17 cells are involved in liver damage through multiple pathways, exacerbating the development of NAFLD. PPC contribute to the attenuation of liver inflammatory responses in mice with NAFLD that may be involved in changes of the Treg/Th17 cell imbalance and associated cytokines.

\section{Conclusions}

Our results indicate that the imbalance of Treg/Th17 cells plays an important role in the pathological mechanism of NAFLD and that changes of related cytokines might be helpful for the differentiation of Treg/Th17 cells and inflammatory responses. PPC intervention may partially attenuate the inflammatory response by adjusting the imbalance of Th17/Treg cells, thus ameliorating the progression of NAFLD.

\section{Abbreviations}

ALT: Alanine aminotransferase; AST: Aspartate aminotransferase;

CHOL: Cholesterol; HF: High-fat diet; HF + PPC: High-fat diet plus polyene phosphatidylcholine capsules; LPS: Lipopolysaccharide; NAFLD: Non-alcoholic fatty liver disease; NASH: Non-alcoholic steatohepatitis; ND: Normal diet; TG: Triglyceride

\section{Acknowledgements}

The work was supported by the Scientific Research Program of Zhejiang Provincial Department of Health (2011KYB058), the Program of Zhejiang


Zhejiang Province Science and Technology Hall (2012C37088).

\section{Availability of data and materials}

The datasets used and/or analysed during the current study available from the corresponding author on reasonable request.

\section{Authors' contributions}

Conceived and designed the experiments: $\mathrm{BH}, \mathrm{ZC}$. Performed the experiments: BH, LW, MY. Performed the statistical analysis: WX, JJ and DC. Contributed materials: JJ. BH, LW, ZC, YS and DC wrote and revised the paper. All authors read and approved the final manuscript.

Competing interests

The authors declare that they have no competing interests.

\section{Consent for publication}

Not applicable

\section{Ethics approval and consent to participate}

The experimental protocols were established according to the Declaration of Helsinki, and the experiments were approved by the Experimental Animal Ethics Committee of the First Affiliated Hospital of Zhejiang Chinese Medical University.

\section{Publisher's Note}

Springer Nature remains neutral with regard to jurisdictional claims in published maps and institutional affiliations.

\section{Author details}

${ }^{1}$ Laboratory of Digestive Disease, the First Affiliated Hospital of Zhejiang Chinese Medical University, 54,Youdian Road, Hangzhou 310006, Zhejiang Province, People's Republic of China. ${ }^{2}$ Department of Equipment, the Third People's Hospital of Hangzhou, 38,Westlake Road, Hangzhou 310009, People's Republic of China. ${ }^{3}$ The University of Melbourne, Melbourne, VIC 3000, Australia. ${ }^{4}$ Department of Preparation Center, the First Affiliated Hospital of Zhejiang Chinese Medical University, 54,Youdian Road, Hangzhou 310006, Zhejiang Province, People's Republic of China. ${ }^{5}$ Department of Gastroenterology, the Second People's Hospital of Yuhang District of Hangzhou, 80,Anle Road, Yuhang District of Hangzhou 311100, People's Republic of China. 'Department of Laboratory Medicine, First Affiliated Hospital, College of Medicine, Zhejiang University, Hangzhou 310003, Zhejiang, People's Republic of China.

Received: 11 September 2016 Accepted: 12 June 2017

Published online: 24 June 2017

References

1. Yoon HJ, Cha BS. Pathogenesis and therapeutic approaches for nonalcoholic fatty liver disease. World J Hepatol. 2014;6(11):800-11.

2. Fan JG, Farrell GC. Epidemiology of non-alcoholic fatty liver disease in China. J Hepatol. 2009:50(1):204-10.

3. Ding J, Li M, Wan X, Jin X, Chen S, Yu C, et al. Effect of miR-34a in regulating steatosis by targeting PPARa expression in nonalcoholic fatty liver disease. Sci Rep. 2015;5:13729. 
4. Farrell GC, Larter CZ. Nonalcoholic fatty liver disease: from steatosis to cirrhosis. Hepatology. 2006;43(2 Suppl 1):S99-S112.

5. Singh SP, Misra B, Kar SK, Panigrahi MK, Misra D, Bhuyan P, et al. Nonalcoholic fatty liver disease (NAFLD) without insulin resistance: Is it different? Clin Res Hepatol Gastroenterol. 2015;39(4):482-8.

6. Ferreyra Solari NE, Inzaugarat ME, Baz P, De Matteo E, Lezama C, Galoppo M, et al. The role of innate cells is coupled to a Th1-polarized immune response in pediatric nonalcoholic steatohepatitis. J Clin Immunol. 2012; 32(3):611-21.

7. Maina V, Sutti S, Locatelli I, Vidali M, Mombello C, Bozzola C, et al. Bias in macrophage activation pattern influences non-alcoholic steatohepatitis (NASH) in mice. Clin Sci. 2012;122(11):545-53.

8. Tang Y, Bian Z, Zhao L, Liu Y, Liang S, Wang Q, et al. Interleukin-17 exacerbates hepatic steatosis and inflammation in non-alcoholic fatty liver disease. Clin Exp Immunol. 2011;166(2):281-90.

9. Hammerich L, Heymann F, Tacke F. Role of IL-17 and Th17 cells in liver diseases. Clin Dev Immunol. 2011:2011:345803.

10. Thiboutot DM, Layton AM, Eady EA. IL-17: a key player in the P. acnes inflammatory cascade? J Invest Dermatol. 2014;134(2):307-10.

11. Korn T, Bettelli E, Oukka M, Kuchroo VK. IL-17 and Th17 Cells. Annu Rev Immunol. 2009;27:485-517.

12. Zhao L, de Qiu K, Ma X. Th17 cells: the emerging reciprocal partner of regulatory T cells in the liver. J Dig Dis. 2010;11(3):126-33.

13. Ye C, Li WY, Zheng MH, Chen YP. T-helper 17 cell: a distinctive cell in liver diseases. Hepatol Res. 2011;41(1):22-9.

14. Hou L, Jie Z, Desai M, Liang Y, Soong L, Wang T, et al. Early IL-17 production by intrahepatic $T$ cells is important for adaptive immune responses in viral hepatitis. J Immunol. 2013;190(2):621-9.

15. Xie ZH, Wang TJ, Zhao ZZ. [Clinical significance of peripheral CD4 + CD25 + Foxp3+ regulatory $T$ cells in nonalcoholic fatty liver disease patients]. Chin Prev Med. 2012;13(1):62-5 [Article in Chinese].

16. Masarone M, Federico A, Abenavoli L, Loguercio C, Persico M. Non alcoholic fatty liver: epidemiology and natural history. Rev Recent Clin Trials. 2014;9(3): 126-33.

17. Carazo A, Salmeron J. Obesity-related non-alcoholic fatty liver disease (NAFLD): A multifactorial process. Rev Esp Enferm Dig. 2014;106(8):501-4.

18. Su TH, Kao JH, Liu JC. Molecular mechanism and treatment of viral hepatitis-related liver fibrosis. Int J Mol Sci. 2014:15(6):10578-604.

19. Jovicic N, Jeftic I, Jovanovic I, Radosavljevic G, Arsenijevic N, Lukic ML, et al. Differential immunometabolic phenotype in Th1 and Th2 dominant mouse strains in response to high-Fat feeding. PLoS One. 2015;10(7):e0134089.

20. Ogura H, Murakami M, Okuyama Y, Tsuruoka M, Kitabayashi C, Kanamoto M, et al. Interleukin-17 promotes autoimmunity by triggering a positivefeedback loop via interleukin-6 induction. Immunity. 2008;29(4):628-36.

21. Rocha AM, Souza C, Rocha GA, de Melo FF, Clementino NC, Marino MC, et al. The levels of IL-17A and of the cytokines involved in Th17 cell commitment are increased in patients with chronic immune thrombocytopenia. Haematologica. 2011;96(10):1560-4.

22. Zhan $\mathrm{YT}$, An W. Roles of liver innate immune cells in nonalcoholic fatty liver disease. World J Gastroenterol. 2010;16(37):4652-60.

23. Luo Z, Wang H, Wu Y, Sun Z, Wu Y. Clinical significance of IL-23 regulating IL-17A and/or IL-17 F positive Th17 cells in chronic periodontitis. Mediators Inflamm. 2014;2014:627959.

24. Mas E, Danjoux M, Garcia V, Carpentier S, Segui B, Levade T. IL-6 deficiency attenuates murine diet-induced non-alcoholic steatohepatitis. PLoS One. 2009; $4(11): e 7929$

25. Zhou L, Lopes JE, Chong MM, Ivanov II, Min R, Victora GD, et al. TGF-betainduced Foxp3 inhibits $\mathrm{T}(\mathrm{H}) 17$ cell differentiation by antagonizing RORgammat function. Nature. 2008;453(7192):236-40.

26. Maggio R, Viscomi C, Andreozzi P, D'Ettorre G, Viscogliosi G, Barbaro B, et al. Normocaloric low cholesterol diet modulates Th17/Treg balance in patients with chronic hepatitis C virus infection. PLoS One. 2014;9(12):e112346.

27. Wang $G Q$, Yang $C L$, Yue DF, Pei $L H$, Zhong $H$, Niu JX. The changes and its significance of Th17 and Treg cells and related cytokines in patients with tuberculosis pleurisy. Allergy Asthma Clin Immunol. 2014;10(1):28.

28. Baier JL, Mattner J. Mechanisms of autoimmune liver disease. Discov Med. 2014;18(100):255-63.

29. Fabbrini E, Cella M, McCartney SA, Fuchs A, Abumrad NA, Pietka TA, et al. Association between specific adipose tissue CD4+ T-cell populations and insulin resistance in obese individuals. Gastroenterology. 2013, 145(2):366-74. e1-3.
30. Hou X, Song J, Su J, Huang D, Gao W, Yan J, et al. CD4(+)Foxp3(+) Tregs protect against innate immune cell-mediated fulminant hepatitis in mice. Mol Immunol. 2015;63(2):420-7.

31. Lv H, Pan Z, Hu S, Chen Y, Zhuang Q, Yao X, et al. Relationship between CD4 + CD25 + Treg cells, Th17 cells and IL-6 and the prognosis of hepatitis $B$ virus-related acute-on-chronic liver failure: a meta-analysis. Chin J Hepatol. 2014;22(7):493-8.

32. Liu Y, She W, Wang F, Li J, Wang J, Jiang W. 3, 3'-Diindolylmethane alleviates steatosis and the progression of NASH partly through shifting the imbalance of Treg/Th17 cells to Treg dominance. Int Immunopharmacol. 2014:23(2):489-98.

\section{Submit your next manuscript to BioMed Central and we will help you at every step:}

- We accept pre-submission inquiries

- Our selector tool helps you to find the most relevant journal

- We provide round the clock customer support

- Convenient online submission

- Thorough peer review

- Inclusion in PubMed and all major indexing services

- Maximum visibility for your research

Submit your manuscript at www.biomedcentral.com/submit
C) Biomed Central 